EPJ Web of Conferences 92,02120 (2015)

DOI: $10.1051 /$ epjconf/ 20159202120

C Owned by the authors, published by EDP Sciences, 2015

\title{
One-dimensional velocity profiles in open-channel flow with intense transport of coarse sediment
}

\author{
Štěpán Zrostlík ${ }^{1, a}$, Vojtěch Bareš ${ }^{1}, J_{a n}$ Krupička ${ }^{1},{\text { Tomáš } \text { Picek }^{1} \text {, and Václav Matoušek }}^{1}$ \\ ${ }^{I}$ Department of Hydraulics and Hydrology, Czech Technical University in Prague, 16629 Prague 6
}

\begin{abstract}
The paper deals with laboratory experiments in open-channel flows with intense transport of model sediment (coarse plastic particles) in our new tilting flume. The major objectives of the paper are: 1 . to discuss applied measuring methods, 2. to analyze measured velocity profiles. Ad 1. A profile of the longitudinal component of local velocity was measured across the vertical axis of symmetry of a flume cross section using three independent measuring methods (Prandtl tube, Ultrasonic Velocity Profiler, Acoustic Doppler Velocity Profiler). Due to strong stratification of the flow in the flume, parts of the profile are measured in regions of very different local concentrations of sediment (from virtually zero concentration to the maximum concentration of bed packing). This makes measurements complicated, particularly for ultrasonic measuring techniques. Profiles measured using the different techniques are evaluated and mutually compared. Ad 2. The layered character of the flow causes that shapes of velocity profiles tend to be different in the transport layer (rich on transported particles) above the bed and in the solids-free region between the top of the transport layer and the water surface. Shapes of the profiles are analyzed. Particular attention is paid to the logarithmic profile in the solids-free region of the flow cross section. The profile can be handled using the law of the hydraulically-rough wall. In the law, the eroded top of the bed with the transport layer is supposed to be the rough boundary and appropriate values are sought for its variables.
\end{abstract}

\section{Introduction}

Intense transport of coarse sediment develops in flow with free water surface if the energy grade line of the flow is steep and the flow produces high bed shear stress. This results in a development of a layer several sediment grains thick through which the sediment is transported at high mean delivered concentration. The layer is called a shear layer and the transport regime the upper plane bed (UPB) regime. Typically, such conditions can be found on surf beaches in an interaction with oscillatory flow of sea waves or in rivers at flood discharges. The last application is of our particular interest. An understanding of friction- and transport conditions is crucial for successful modeling and prediction of this complex type of flow. So far, research results and publications on this subject are limited and understanding of the processes insufficient. A majority of the work has been done for pressurized flows in enclosed profiles [1-7]. Examples of experimental and analytical works for intense transport of sediment in open channels are [8-11]. In the literature, there is a general agreement that the log-law for a hydraulically rough boundary

\footnotetext{
${ }^{\mathrm{a} C}$ Corresponding author: stepan.zrostlik@fssv.cvut.cz
}

$$
\sqrt{\frac{8}{\lambda_{b}}}=\frac{U}{u_{b}^{*}}=2.5 \ln \left(B_{r} \frac{R_{b}}{k_{s}}\right),
$$

can be applied in the UPB regime. In Eq. (1), $\lambda_{b}=$ bed friction coefficient, $u_{b} *=$ shear velocity associated with channel bed, $U=$ average velocity in the cross section of flow above bed, $B_{r}=$ integration constant (for open channel $\left.B_{r}=11.1\right), R_{b}=$ hydraulic radius associated with bed and $k_{s}=$ bed roughness. In this law, an evaluation of the bed roughness seems to be different for the so-called weak-transport condition (characterized by a certain threshold value of the Shields parameter) and the intense transport condition [4-5]. For the intense-transport condition, the roughness depends on the Shields parameter and hence a solution for the friction coefficient is implicit which leads to computation failures in some cases. Recently, an explicit formula has been proposed [10]. However, the formula is empirical and a more general solution is sought. A knowledge of local velocity distribution in flow with a shear layer may contribute to better understanding of mechanisms governing friction and transport of sediment. A general formula for the distribution of local velocity in the boundary layer above the hydraulically rough boundary predicts a logarithmic velocity profile, 


$$
\frac{u}{u_{b}^{*}}=2.5 \ln \left(B \frac{y}{k_{s}}\right) \text {, }
$$

Eq. (1) is derived from this Eq. (2) in which $B=30$ and $y=$ distance from the boundary in the direction perpendicular to the boundary. Our objective is to measure velocity profiles for $u(y)$ in the flows carrying sediments in the UPB regime using three independent methods and compare them with the log law (Eq. 2).

\section{Methods}

\subsection{Experimental rig}

Experiments were carried out in an experimental rig in the Water Engineering Laboratory of the Czech Technical University (CTU) in Prague. The rig is a tilting flume with a recirculating system (Figure 1). The system is composed of two parts, one for recirculating sedimentwater mixture and the other for recirculating water only. The mixture part includes a vertical U-loop for continuous measurement of differential pressures (Rosemount, DP1151) in both (ascending and descending) legs of the loop. From the DP measurements the mean delivered concentration of sediment in the recirculating mixture can be determined [12] and continuously monitored. Both mixture- and water parts are equipped with electromagnetic flow meters (Primo TCM142/02-3715 for flow of water; KROHNE OPTIFLUX 5000 for flow of mixture) and centrifugal pumps with the variable frequency drive. The flume is 8 $\mathrm{m}$ long, $0.2-\mathrm{m}$ wide and $0.25-\mathrm{m}$ deep. It can be tilted from the horizontal position to the maximum slope of 16.7 degrees. There is a $1-\mathrm{m}$ long inlet section at the beginning of the flume with distributors of flow. At the outlet of the flume, a 1-m long outlet box takes care of flow energy dissipation and directs the flow of mixture in the separation tank below the box.

In the flume, four ultrasonic transducers (Pepperl-Fuchs, UC500-30GM-IUR2-V15) measure positions of water surface at distances 2.3, 3.3, 4.8, $6.3 \mathrm{~m}$ behind the flume inlet. Positions of the top of sediment deposit are observed visually through the glass side walls of the flume.

\subsection{Tested sediment and flow conditions}

Model sediment is a fraction of plastic grains of an ellipsoidal shape and a very narrow grain size distribution (commercial name: Hostaform). We measured the following properties of the sediment: equivalent diameter $d=3.18 \mathrm{~mm}$, density $\rho_{s}=1359 \mathrm{~kg} \cdot \mathrm{m}^{-3}$, terminal settling velocity $w_{t}=0.131 \mathrm{~ms}^{-1}$, and loose-poured bed concentration $C_{b}=0.6$.

In total, we collected 66 test runs with this sediment in our rig. In 32 runs, velocity profiles were measured additionally to the integral quantities of flow. Steadystate conditions and virtually uniform flow were maintained during the tests. The runs covered a broad range of the measured parameters: the total discharge 4.6-19 $1 / \mathrm{s}$, the flow depth $39-115 \mathrm{~mm}$, the delivered concentration of sediment $1-25 \%$, and the slope of the energy grade line 0.31-3.32 deg. An analysis of the integral data revealed rather different behavior and trends in the observed flows in regions separated by the threshold value of the Shields parameter $\Theta_{t h} \approx 1.1$ [10]. In Figure 2, the change in a trend is demonstrated on the relationship between the bed roughness and the Shields parameter. The relative roughness starts to increase with the increasing $\Theta$ when $\Theta$ passes the threshold value $\Theta_{t h}$.

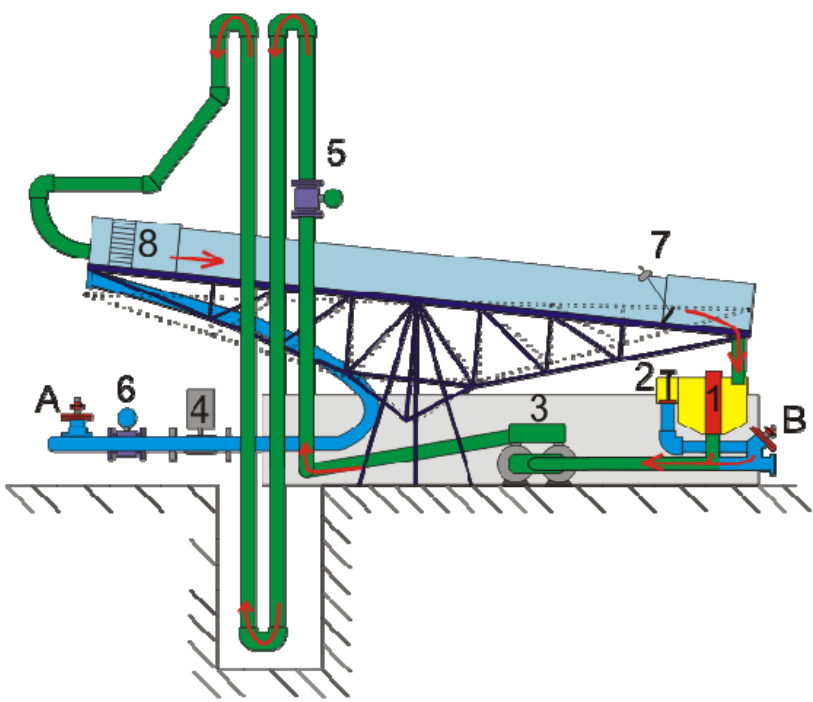

Figure 1. Experimental rig. Legend: 1-supply and separation tank; 2 - overflow; 3 - centrifugal pump for mixture; 4 centrifugal pump for water; 5,6 - flow meters; 7 - outlet with overshot weir; 8- inlet with flow distributors; A,B - valves

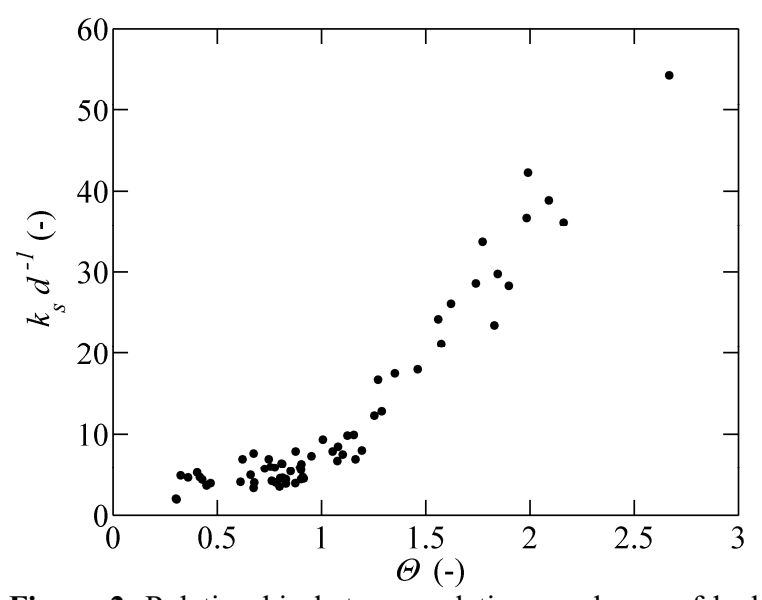

Figure 2. Relationship between relative roughness of bed and Shields parameter

\subsection{Flow structure}

Figure 3 shows a typical layered structure of the tested flows. Basically, the flow is composed of two layers. Below the water surface, there is a layer of clean water and occasionally saltating particles. Beneath this layer, the shear layer occurs with the concentration of moving sediment increasing with the depth. The shear layer is adjacent to the sediment deposit in which grains do not 
move at all. The thickness of the individual layers varies considerably with the change of discharge and/or bed slope.

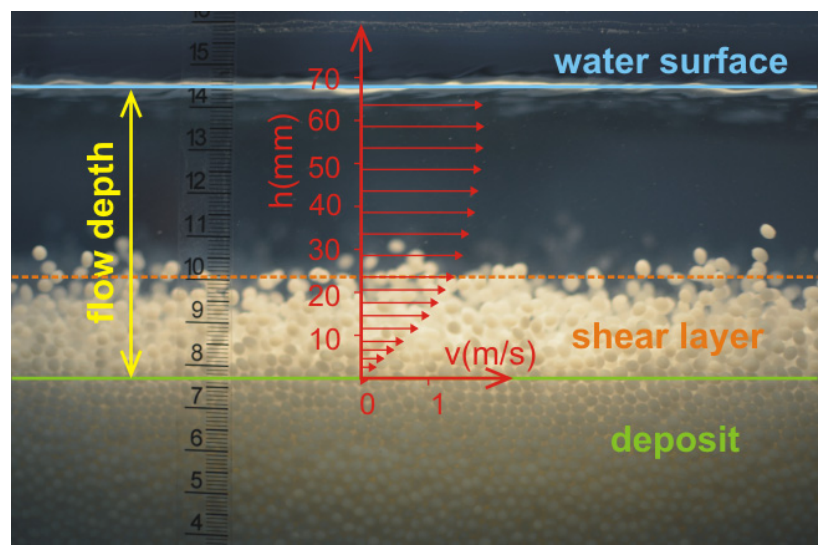

Figure 3. Schematic structure of flow and velocity profile of water.

\subsection{Local velocity measurement}

Three independent methods were used to measure velocity profiles above the deposit. All instruments were located to the same measuring location: to the center of the flow cross section $4.2 \mathrm{~m}$ behind the flume inlet.

Each applied method (and instrument) has its limitations and its validity must be evaluated using specific criteria for particular conditions in the tested flows.

\subsubsection{Prandtl tube (PT)}

PT was connected to a differential pressure transducer (Siemens, SITRANS P, range $0.6 \mathrm{~m}$ ). A collected signal from the transducer contained 4500 samples at the frequency of $100 \mathrm{~Hz}$. The outer diameter of PT was $6 \mathrm{~mm}$ and the diameter of the frontal hole was $1.5 \mathrm{~mm}$ (Figure $4 a)$. The step in vertical positions of two measured points was 5 or $4 \mathrm{~mm}$ in the water layer above the shear layer and 3 or $2 \mathrm{~mm}$ within the shear layer. The step was dependent on the depth of flow and the thickness of the shear layer.

One limitation for using PT was given by the diameter of PT: the highest measured point was $3 \mathrm{~mm}$ under the water surface if the surface did not fluctuate considerably. The other limitation was given by the sensitivity of the pressure transducer which did not allow to sense accurately local velocities below say $10 \mathrm{cms}^{-1}$.

\subsubsection{Ultrasonic velocity profiler (UVP)}

As PT, UVP (Takeda, 1997) was used to measure only the local longitudinal velocity component of flow. A $4 \mathrm{MHz}$ transducer was directed against the flow using a support (Figure 4c). The outer diameter of the transducer was $8 \mathrm{~mm}$ and the diameter of the active area was $5 \mathrm{~mm}$. The velocity was evaluated at the anti-stream wise longitudinal distances of $14.5,33$ and $44.5 \mathrm{~mm}$ in front of the transducer. The vertical positions for the measuring points were chosen the same as for PT. One point of a velocity profile was evaluated from 1500 samples at the frequency of $60 \mathrm{~Hz}$. The UVP probe tended to produce slightly different results for the three different longitudinal distances. A comparison with PT showed that the UVP results for the distances of 33 and $44.5 \mathrm{~mm}$ agreed well with PT in the water layer. In the shear layer, the agreement was satisfactory for the distance of 44.5 $\mathrm{mm}$.

\subsubsection{Acoustic Doppler Velocity Profiler (ADVP)}

ADVP (NORTEK SA, Vectrino Profiler) detects a velocity profile in a 32-mm thick part of the flow depth in one time. The vertical resolution of the measurement is 1 mm. A major advantage of ADVP is its ability to measure local velocities at different vertical positions at once, its disadvantage is that it is not applicable for shallow flows (Figure 4b). Furthermore, the shape of the probe receivers poses a problem as in case of small submergence the receivers attract air pockets which gather in the wakes behind receivers and may influence measurements.

The processing of ADVP data included filtering out data with a low correlation of the signal and checking whether the sufficient number of data remained available for further processing after the filtration.

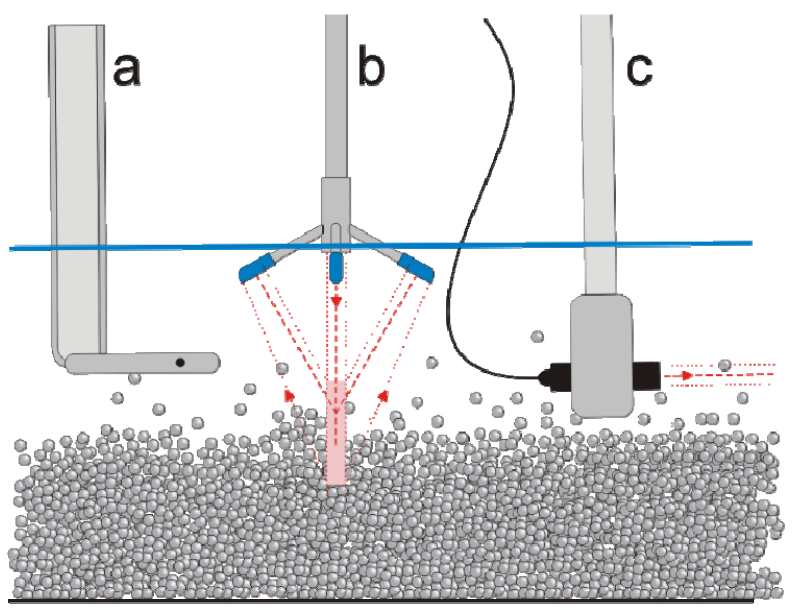

Figure 4. Measuring instruments: $a-$ Prandtl tube; $b-A D V$ Profiler; c - UVP

\section{Evaluation of Velocity Profiles}

\subsection{Logarithmic profile for water flow}

In order to verify a presence of the theoretical logarithmic profile in water flow above rough bed in our flume and to check an ability of the applied measuring methods to sense the profile, one test run was carried out for flow of water above deposit with no transport of sediment.

Glass beads of 3-mm size were used to form deposit in the flume. The flow was set to a value of the Shields parameter below the critical value for incipient motion of grains. Figure 5 summarizes the results for PT and ADVP and compares them with the logarithmic profile using

$$
\frac{u}{u_{b}^{*}}=2.5 \ln \left(B \frac{y-y_{i n i}}{k_{s}}\right),
$$


in which $y_{i n i}=$ vertical displacement of the profile origin with respect to the position of the flow boundary. For the profile in Figure $5, u_{b} *=0.044 \mathrm{~m} / \mathrm{s}$ is determined from the measured integral parameters; $k_{s}=2.3 \mathrm{~mm}$; and $y_{i n i}=2.5 \mathrm{~mm}$. The logarithmic profile agrees well with the measured velocity profiles except for the highest positions near the water surface.

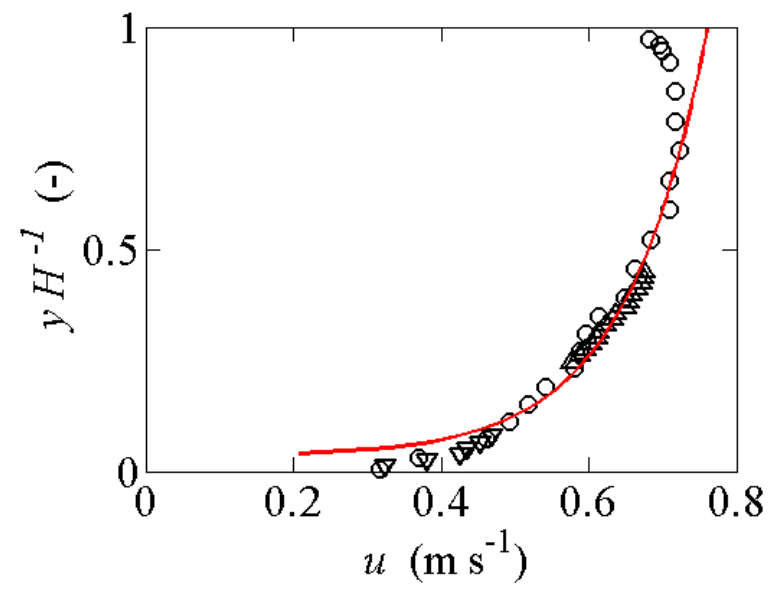

Figure 5. Logarithmic profile in water flow above the rough bed with no transport of sediment. Legend: line - theoretical logarithmic profile using Equation 3: circles - measurements using PT, triangles - measurements using ADVP

\subsection{Composite profile for mixture flow}

For the flow carrying sediment in the shear layer we assume that the log-profile may be present in at least a part of the water layer above the shear layer but within the shear layer itself it needs to be replaced with a profile of some other shape. As a first approximation we assume a power-law shape represented by the equation:

$$
\frac{u}{u_{t r}}=\left(\frac{y-\Delta y}{y_{t r}-\Delta y}\right)^{n},
$$

in which $u_{t r}=$ local velocity at the position $y_{t r}$ where the power profile smoothly transforms to the logarithmic profile; $\Delta y=$ position of zero velocity (i.e. the displacement of the origin of the power-law profile); $\mathrm{n}=$ power-law exponent. A schematic composition of the composite profile is shown on the right-hand side of Figure 6. On the left-hand side of Figure 6, the shape of a schematic concentration profile is given.
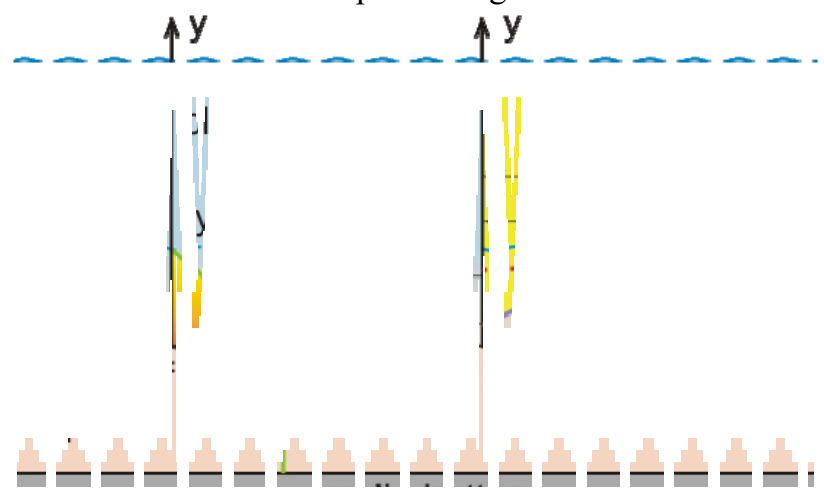

Figure 6. Schematic structure of flow with shear layer

\subsection{Optimization of composite profile parameters}

For each of the 32 collected profiles, an optimization procedure was employed to find values for the parameters in Equations 3 and 4 to produce the best fit to the measured velocity profiles. The procedure was based on the least square method. A comparison of the profiles sensed by the three measuring methods allowed us to select reliable data which were used as inputs to the optimization procedure.

At first, the optimization was performed for all available parameters $\left(u_{b}^{*}, k_{s}, y_{i n i}, y_{t r}, \Delta y, n\right)$. However, it appeared that much clearer results were achieved when the number of optimized parameters had been reduced. Therefore, it was decided to keep the profile in the shear layer linear $(n=1)$ and to use avalue of the bed shear velocity (determinedfrom the measured integral quantities) as an input to the procedure. Our choice of the linear profile was based on preliminary processing of our profiles and also on experience with velocity profiles measured at very similar conditions by Capart and Fraccarollo [9].

Observed shapes of velocity profiles seem to support the earlier finding that flows are rather different at $\Theta<\Theta_{t h}$ than at Shields parameters higher than the threshold value $\Theta_{t h}=1.1$. Figure 7 shows a typical profile for flow at $\Theta<\Theta_{t h}(\Theta=0.42)$. In the flow, the water layer with a log-profile (white area) dominates over a much thinner shear layer with a linear profile (grey area). The interface between linear and logarithmic profile (i.e. the position of $y_{t r}$ ) is indicated by the dotted line. The dash line indicates the position of the origin of the logarithm profile $\left(y_{i n i}\right)$. Measured local velocities used in the optimization procedure are indicated by black points in Figure 7.

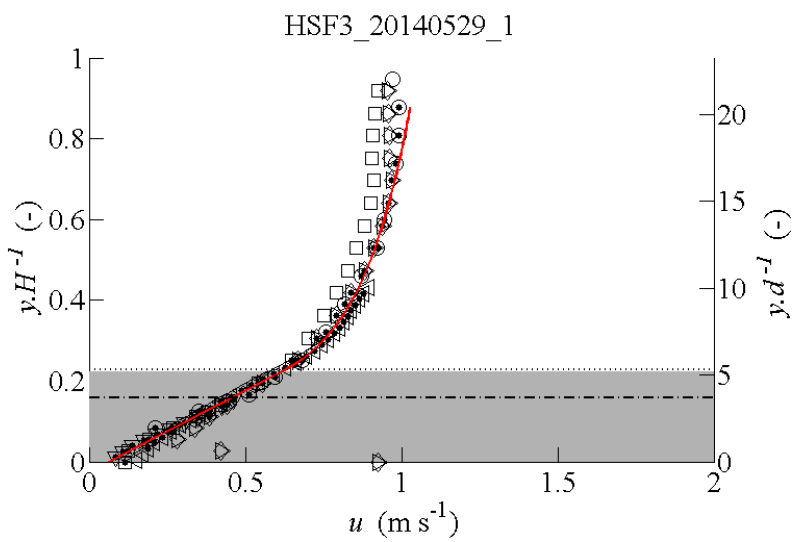

Figure 7. Optimized velocity profile $(\Theta=0.42)$. Legend: dotted line - position of transition in composite profile, dash line position of origin of log profile, grey area - shear layer; local velocity symbols: circle - PT; squares, triangles to right and diamonds - UVP; triangles to left and down - ADV; black points - data used for optimization

Figure 8 shows a typical profile for flow with intense transport of sediment at $\Theta>\Theta_{t h}(\Theta=1.99)$. The shear layer occupies much bigger portion of the total depth of flow. 


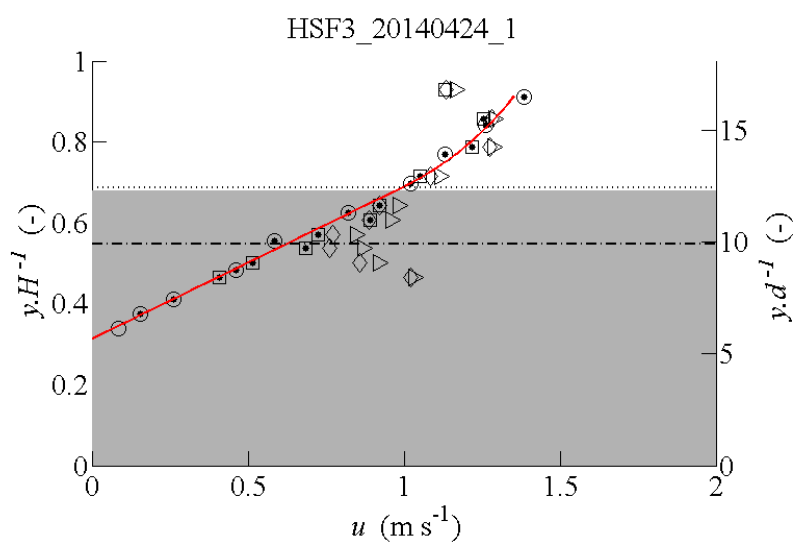

Figure 8. Optimized velocity profile $(\Theta=1.99)$ Legend: 1 dotted line - position of transition in composite profile, dash line - position of origin of log profile, grey area - shear layer; local velocity symbols: circle - PT, squares triangles and diamonds - UVP

Figures 9-16 summarize results of the optimization procedure. In the figures, crosses represent profiles for $\Theta<\Theta_{\text {th }}$ (weak transport of sediment) and circles the rest of the profiles (intense transport of sediment).

Figure 9 demonstrates how the vertical position of the interface between the linear profile and the log profile shifts towards the water surface with the increasing hydraulic radius associated with bed, $R_{b}$. Both heights are normalized by the depth of flow, $H$. Increasing bed shear causes an increase in the portion of the flow depth influenced by bed resistance resulting in the linear profile. It is interesting to see that the increase reaches its maximum at $\Theta_{t h}$ showing that $y_{t r} / H \approx 0.7$ for $\Theta>\Theta_{t h}$. This implies that the linear profile dominates at high bed shear associated with intense transport, but there is always a certain portion of the total u-profile that can be considered logarithmic.

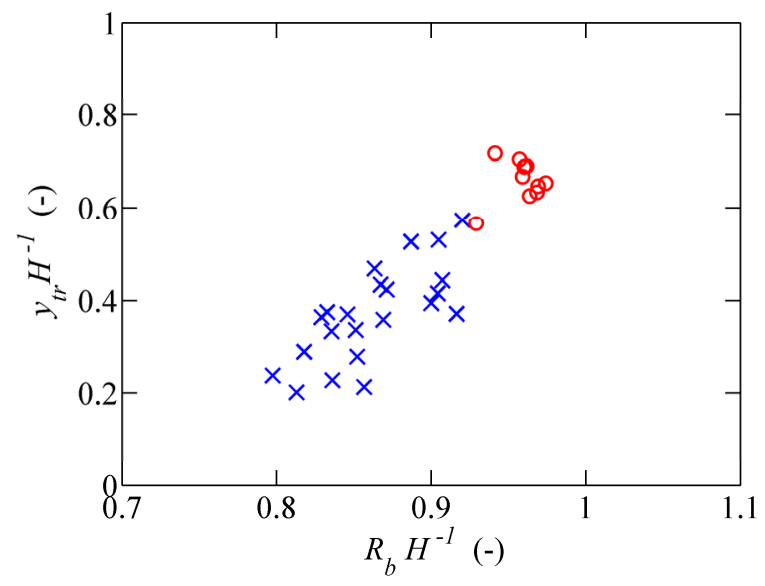

Figure 9. Relation between position of transition in composite profile and hydraulic radius; both parameters normalized by flow depth.

In Figure 10, the position of the interface is related to the position of the top of the shear layer $\left(H_{s h}\right.$, determined visually just like the top of the deposit) and to the Shields parameter. At $\Theta<\Theta_{t h}$, the correlation is weak perhaps due to big uncertainty in reading out positions for very thin shear layers. At $\Theta>\Theta_{t h}$, the correlation is tighter and suggests that, in principle, the position of the interface in the composite profile corresponds with the top of the shear layer, at least for very intense transport, $\Theta>>\Theta_{t h}$.

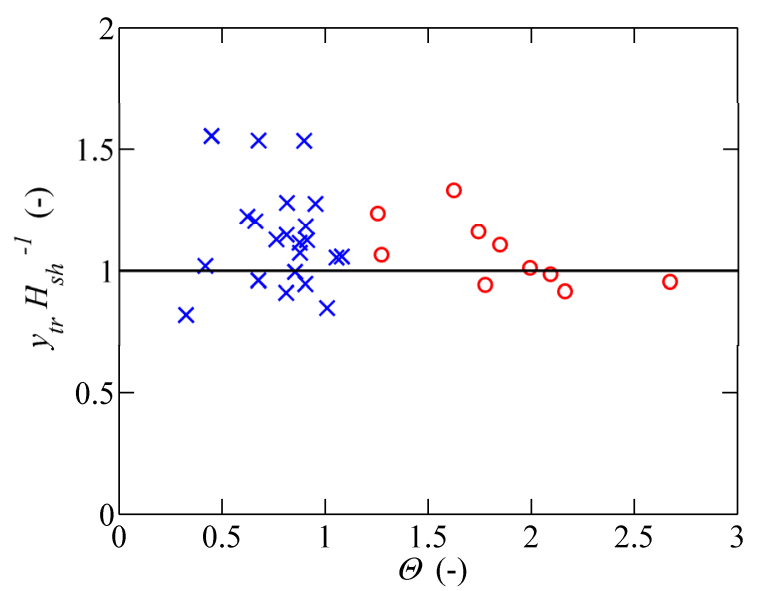

Figure 10.Relation between position of transition in composite profile normalized by position of the top of shear layer and Shields parameter.

\subsection{Linear part of velocity profile}

Three parameters $\left(\Delta y, y_{t r}\right.$ and $\left.u_{t r}\right)$ were optimized in the equation of a linear part of the composite profile.

Figure 11 reveals that the position of the origin of the linear profile is quite sensitive to the Shields parameter and may reach displacement bigger than two grain sizes for intense-transport conditions. Certainly, there is some uncertainty in read-outs of the visually observed positions of the top of the deposit that may obscure the results (we are talking here about the resolution in the order of magnitude of one grain size). However, the trend of an increase of the origin with the Shields parameter is there. In fact, closer visual observations in the region very near the deposit, where the measuring methods cannot provide sufficiently accurate measurements, suggest that there might be a tail in the u-profile as detected by measurements in [11].

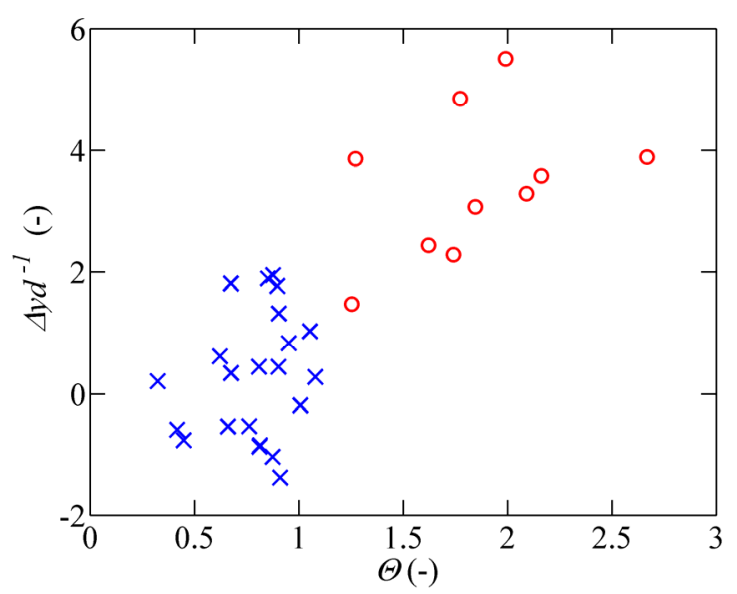

Figure 11. Relation between position of origin of linear profile normalized by grain size and Shields parameter. 
The literature suggests that the value of the ratio of the velocity at the top of the shear layer and the bed shear velocity does not vary with the Shields parameter. Despite considerable scatter in data shown in Figure 12, our resultsdo not differ much from the value of 9.4 suggested in $[7,13]$.

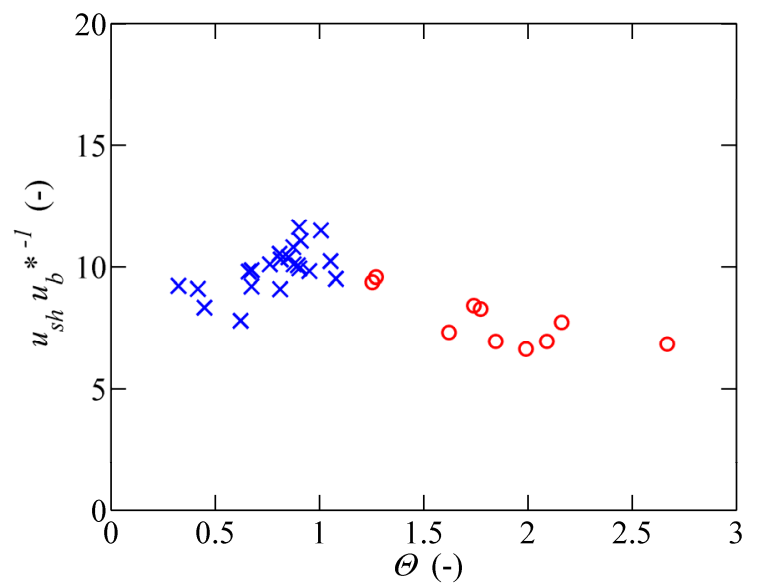

Figure 12.Relations between local velocity at top of shear layer normalized by bed shear velocity and Shields parameter.

Another parameter describing the linear part of a $\mathrm{u}-$ profile is its gradient. It is defined as the change of velocity (from 0 to $u_{t r}$ ) over the height of the linear profile,

$$
\frac{d u}{d y}=\frac{u_{t r}}{y_{t r}-\Delta y} .
$$

Figure 13 shows that for $\Theta<\Theta_{t h}$, the gradient increases with the Shields parameter. At $\Theta>\Theta_{t h}$, however, the gradient remains virtually constant.

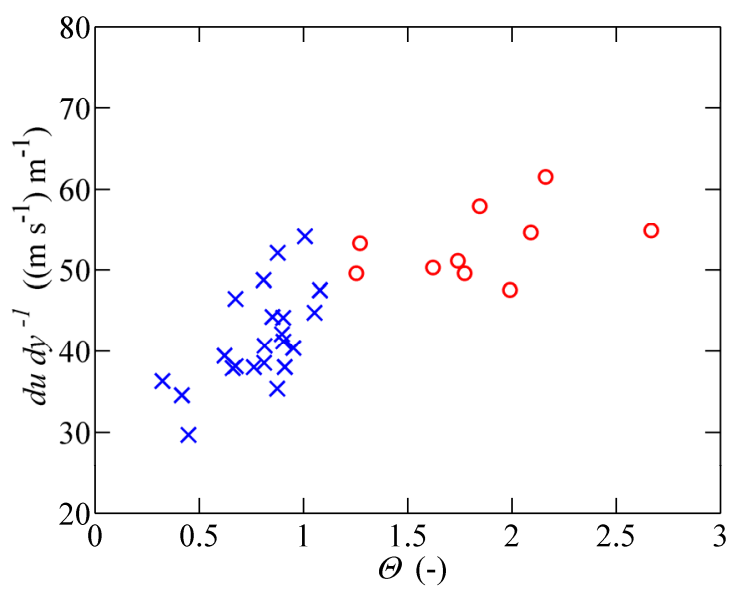

Figure 13. Relation between gradient of linear profile and Shields parameter.

\subsection{Logarithmic part of velocity profile}

The log part is smoothly connected to the linear part (meaning that their gradients equal at $y_{t r}$ ) of the composite profile. Two parameters were optimized in the log law (Equation 3): the roughness, $k_{s}$, and the position of the origin of the log profile, $y_{\text {ini }}$.

Figure 14 shows the dimensionless position of the origin within the visually observed shear layer plotted against the Shields parameter. In the literature [7], a value of 0.56 is reported and it is in a reasonable agreement with our results for $\Theta>\Theta_{t h}$.

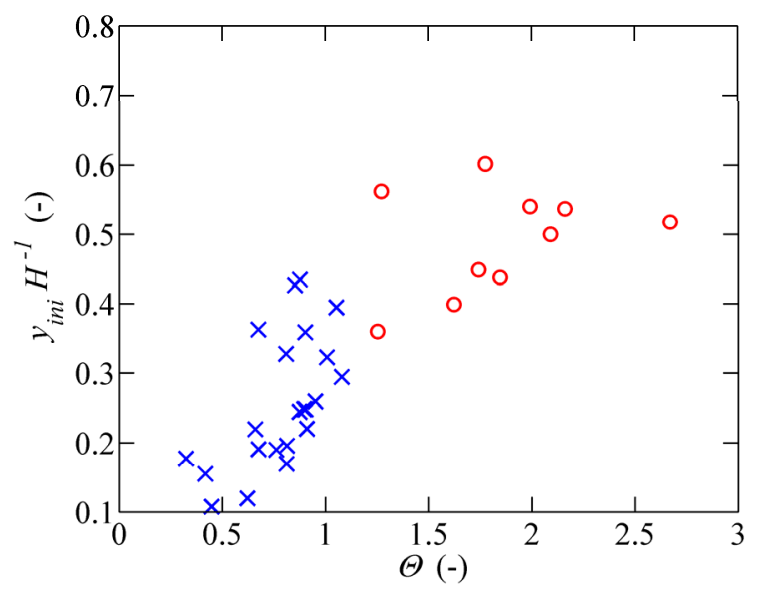

Figure 14. Position of origin of log-profile normalized by thickness of shear layer and related to Shields parameter.

At high bed shear, the bed roughness should be related to the thickness of the shear layer. An analysis in [7] suggests that the roughness equals to approximately one third of the height of the shear layer. Our results (Figure $15)$ indicate that the ratio $k_{s} / H_{s h}$ may be sensitive to the Shields parameter, but values do not differ too much from the suggestion in [7].

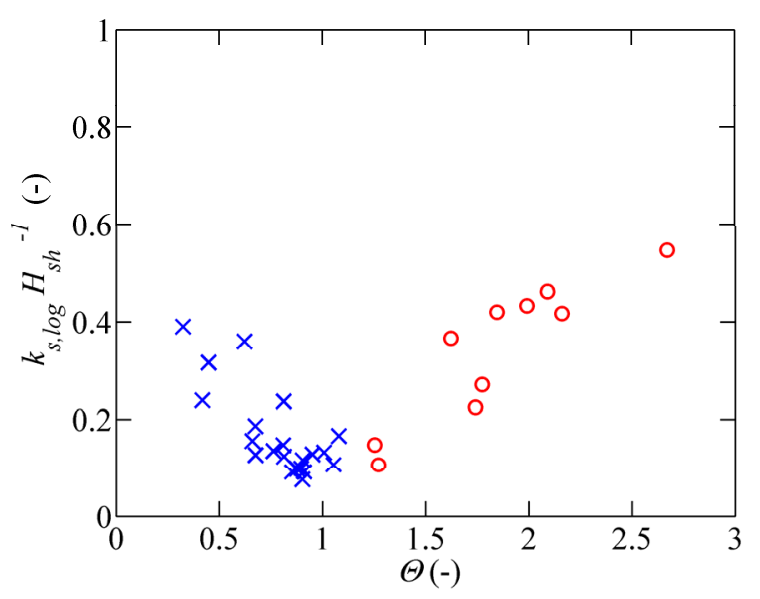

Figure 15. Bed roughness normalized by height of shear layer and related to Shields parameter.

Figure 16 is the same plot as Figure 2 but for values of the bed roughness are determined differently. While the roughness is determined using the friction formula (Equation 1) in Figure 2, the same parameter is determined from the velocity-profile formula (Equation 3 ) in Figure 16. The absolute values are different (the optimization of the log-profile produces lower values of 
the roughness than the processing using the integral parameters) but the trends agree.

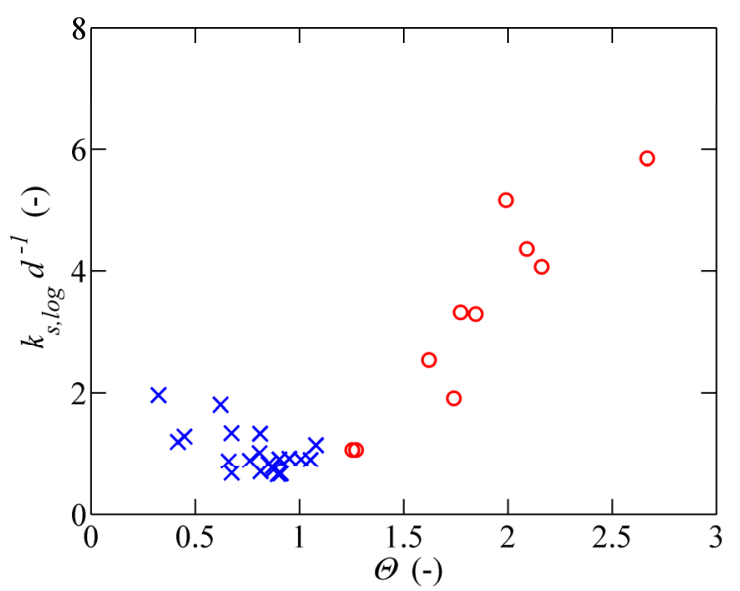

Figure 16. Bed roughness from log-profile normalized by grain size and related to Shields parameter.

\section{Conclusions}

The study presents results of measurements of velocity profiles in open-channel flows with intense transport of plastic particles in the upper plane bed regime.

In the observed layered flow, the velocity profiles seem to be composed of two parts, the logarithmic in the upper particle-arm layer and linear in the lower particle-rich layer. The optimization procedure reveals that the transition between the two parts in the composite profile approximately corresponds with the top of the shear layer.

The bed roughness determined using the velocity profiles exhibits the same trend as the bed roughness determined using the friction law (and hence integral quantities) - it tends to increase considerably with the Shields parameter if intense transport of sediment is involved. However, values of the roughness determined by the two different methods are different.

Tests with another model sediments will be carried out to confirm the phenomena and trends observed for the plastic sediment in our tilting flume.

\section{Acknowledgments}

The research has been supported by the Czech Science Foundation through the grant project No. P105/12/1082 and by Faculty of Civil Engineering of the Czech Technical University in Prague through the student grant project No. SGS14/179/OHK1/3T/11.

\section{References}

1. Matoušek,V., J. Hydraul. Eng.-ASCE,135 (12), 1101-1106, (2009)

2. Matoušek,V. \& Krupička, J. Hydrol. Hydromech., 57(3), 191-199 (2009)
3. Matoušek, V. Particul. Sci. Technol., 29(1), 89-106. (2011).

4. Matoušek, V., Krupička, J. \& Picek, T., J. Hydrol. Hydromech., 61(2), 120-125, (2013)

5. Matoušek, V. \& Krupička, J., P. I. Civil Eng.-Mar. $E n .$, (to be published)

6. Sumer et al., J. Hydraul. Eng.-ASCE,117, (1996)

7. Pugh, F. J. \& Wilson, K. C., J. Hydraul. Eng.ASCE, 117, (1999)

8. Smart, G. M., J. Hydraul. Eng.-ASCE,106, (1984)

9. Capart, H. \& Fraccarollo, L., Geophysi. Res. Lett., 38, (2011)

10. Matoušek, V. \& Krupička, J., Proc. River Flow 2014, pp. 917-922.

11. Revil-Baudard,T., Chauchat, D. \& Barthélémy, E., River Flow 2014, (2014)

12. Matoušek, V., Settling Slurries Advanced Topics. Proc. 37th Annual Course on Transportation of Solids Using Centrifugal Slurry Pumps, GIW Industries and Georgia Regents University, Augusta, GA, USA (2014)

13. Wilson, K. C., J. Hydraul. Eng.-ASCE,115(6), 825830, (1989) 